\title{
Observability of the General Relativistic Precession of Periastra in Exoplanets
}

\author{
Andrés Jordán ${ }^{1,2}$ and Gáspár Á. Bakos ${ }^{1}$ \\ ${ }^{1}$ Harvard-Smithsonian Center for Astrophysics, 60 Garden St., Cambridge MA 02138, USA \\ email: ajordan@cfa.harvard.edu, gbakos@cfa.harvard.edu \\ ${ }^{2}$ Departamento de Astronomía y Astrofísica, Pontificia Universidad Católica de Chile, Casilla \\ 306 , Santiago 22, Chile.
}

\begin{abstract}
The general relativistic precession rate of periastra in close-in exoplanets can be orders of magnitude larger than the magnitude of the same effect for Mercury. The realization that some of the close-in exoplanets have significant eccentricities raises the possibility that this precession might be detectable. We explore here the observability of the periastra precession using radial velocity and transit light curve observations. Our analysis is independent of the source of precession, which can also have significant contributions due to additional planets and tidal deformations. We find that precession of the periastra of the magnitude expected from general relativity can be detectable in timescales of $\lesssim 10$ years with current observational capabilities by measuring the change in the primary transit duration or in the time difference between primary and secondary transits. Radial velocity curves alone would be able to detect this precession for super-massive, close-in exoplanets orbiting inactive stars if they have $\sim 100$ datapoints at each of two epochs separated by $\sim 20$ years. The contribution to the precession by tidal deformations may dominate the total precession in cases where the relativistic precession is detectable. Studies of transit durations with Kepler might need to take into account effects arising from the general relativistic and tidal induced precession of periastra for systems containing close-in, eccentric exoplanets. Such studies may be able to detect additional planets with masses comparable to that of Earth by detecting secular variations in the transit duration induced by the changing longitude of periastron.
\end{abstract}

\section{Introduction}

By virtue of their small semi-major axes and high eccentricities, the longitude of periastron $\omega$ of some of the newly discovered exoplanets are expected to precess due to General Relativistic (GR) effects at rates of degrees per century. This is orders of magnitude larger than the same effect observed in Mercury in our Solar System (43" / century), which offered one of the cornerstone tests of GR. Furthermore, the massive, close-in eccentric planets induce significant reflex motion of the host star, therefore enhancing the detectability of the precession directly via radial velocities.

We address here the observability of the precession of the longitude of periastron with the magnitude expected from GR in exoplanets using radial velocity and transit timing observations. We also consider the periastra precession due to planetary perturbers and tidal deformations, which can have contributions comparable or greater than that of GR. We refer the reader to Jordán \& Bakos (2008) for more details and to Pál \& Kocsis (2008) for independent work that also explores the measurable effects of the periastra precession induced by GR. 
Table 1. GR precession of exoplanets with $e>0.1$ and GR Precession Rates $>1^{\circ} /$ century

\begin{tabular}{lccccrrrr}
\hline \hline \multicolumn{1}{c}{ Name } & $\begin{array}{c}a \\
(\mathrm{AU})\end{array}$ & $e$ & $\begin{array}{c}M_{\star} \\
\left(M_{\odot}\right)\end{array}$ & $\begin{array}{c}\text { Period } \\
(\text { days })\end{array}$ & $\begin{array}{c}\mathrm{K} \\
\left(\mathrm{m} \mathrm{sec}^{-1}\right)\end{array}$ & $\begin{array}{c}\omega \\
(\mathrm{deg})\end{array}$ & $\begin{array}{c}\dot{\omega}_{\mathrm{GR}} \\
/ \text { century }\end{array}$ & TEP? \\
\hline HD49674 b & 0.058 & 0.290 & 1.060 & 4.944 & 13.7 & 283.0 & 1.576 & 0 \\
HD88133 b & 0.047 & 0.133 & 1.200 & 3.416 & 36.1 & 349.0 & 2.958 & 0 \\
GJ 436 b & 0.028 & 0.159 & 0.410 & 2.644 & 18.7 & 339.0 & 2.234 & 1 \\
HD118203 b & 0.070 & 0.309 & 1.230 & 6.133 & 217.0 & 155.7 & 1.231 & 0 \\
HAT-P-2 b & 0.069 & 0.507 & 1.350 & 5.633 & 884.0 & 184.6 & 1.836 & 1 \\
HD185269 b & 0.077 & 0.296 & 1.280 & 6.838 & 90.7 & 172.0 & 1.046 & 0 \\
XO-3 b & 0.048 & 0.260 & 1.410 & 3.192 & 1471.0 & -15.4 & 3.886 & 1 \\
\hline
\end{tabular}

\section{Expected Precession of Periastra}

\subsection{General Relativistic Precession}

The rate of precession of the longitude of periastron (hereafter denoted by $\omega$ ) due to General Relativity (GR) is given by:

$$
\dot{\omega}_{\mathrm{GR}}=\frac{3.904\left(M_{\star} / M_{\odot}\right)}{(a / 0.1 \mathrm{AU})\left(1-e^{2}\right)(P / \text { day })}[\% / \text { century }] .
$$

We use equation 2.1 to estimate the expected precession for currently known exoplanets as listed in the online California \& Carnegie Catalog of Exoplanets (Butler et al. 2006). We list in Table 1 all known exoplanets that have $e>0.1$ and that have $\dot{\omega}_{\mathrm{GR}}>1^{\circ} /$ century.

\subsection{Stellar Quadrupole, Tides and Additional Planets}

Besides the GR precession mentioned above, $\omega$ can precess due to the presence of additional effects. Miralda-Escudé (2002) and Heyl \& Gladman (2007) discussed the observability of changes in $\omega$ caused by a stellar quadrupole moment and perturbations from other planets. Additionally, tidal deformations induced on the star and the planet can also produce a secular change on $\omega$, an effect not considered in the studies mentioned above. The effect of apsidal motions induced by tidal deformations is a well known effect in eclipsing binaries (Sterne 1939; Quataert et al. 1996; Smeyers \& Willems 2001), and was included in the analysis of the planetary system around HD 83443 by Wu \& Goldreich (2002).

The precession caused by a stellar quadrupole moment is given to second order in $e$ and first order in $\left(R_{*} / a\right)^{2}$ by

$$
\dot{\omega}_{\text {quad }} \approx 0.043\left(\frac{P}{\text { day }}\right)^{-1}\left(\frac{J_{2}}{10^{-6}}\right)\left(\frac{R_{\star}}{R_{\odot}}\right)^{2}\left(\frac{a}{0.1 \mathrm{AU}}\right)^{-2}[\% / \text { century }] .
$$

where $J_{2}$ is the quadrupole moment (Murray \& Dermott 1999).

The tidal deformations induced on the star and the planet will lead to a change in $\omega$ which is given, under the approximation that the objects can instantaneously adjust their equilibrium shapes to the tidal force and considering up to second order harmonic distortions, by

$$
\dot{\omega}_{\text {tide }} \approx 1.6 f(e) \mathcal{T}\left(\frac{P}{\text { day }}\right)^{-1}\left(\frac{k_{2, p}}{0.1}\right)\left(\frac{a}{0.05 \mathrm{AU}}\right)^{-5}\left(\frac{R_{p}}{R_{J}}\right)^{5}\left(\frac{M_{J}}{M_{p}}\right)\left(\frac{M_{\star}}{M_{\odot}}\right) \quad\left[{ }^{\circ} / \text { century }\right],
$$


where $f(e) \equiv\left(1-e^{2}\right)^{-5}\left[1+(3 / 2) e^{2}+(1 / 8) e^{4}\right], k_{2, p}$ and $k_{2, s}$ are the apsidal motion constants of planet and star respectively $\dagger$ (Sterne 1939) and we have introduced $\mathcal{T} \equiv$ $1+\left(R_{\star} / R_{p}\right)^{5}\left(M_{p} / M_{\star}\right)^{2}\left(k_{2, s} / k_{2, p}\right)$, which is $\approx 1$ for the case of a close-in Jupiter.

The precession of the periastra caused by a second planet, which we dub a "perturber", is given to first order in $e$ and lowest order in $\left(a / a_{2}\right)$ by

$$
\dot{\omega}_{\text {perturber }} \approx 29.6\left(\frac{P}{\text { day }}\right)^{-1}\left(\frac{a}{a_{2}}\right)^{3}\left(\frac{M_{\star}}{M_{\odot}}\right)^{-1}\left(\frac{M_{2}}{M_{\oplus}}\right) \quad[\% / \text { century }]
$$

where $M_{2}$ is the mass of the second planet and $a_{2}$ the semi-major axis of its orbit (Murray \& Dermott 1999; Miralda-Escudé 2002).

It is clear from the expressions above that for a close-in Jupiter we have in general $\dot{\omega}_{\text {quad }} \ll \dot{\omega}_{\text {tide }} \sim \dot{\omega}_{\text {perturber }} \sim \dot{\omega}_{\text {GR }}$. Therefore, any detected precession of $\omega$ is expected to arise by GR, the effect of additional planets or tidal deformations.

\section{Observability of Periastra Precession in Extra-Solar Planets}

In what follows we will discuss the observability of changes in $\omega$ using radial velocity and transit observations. The discussion that follows addresses the detectability of changes in $\omega$ independent of their origin.

\subsection{Duration of Transits}

The logarithmic derivative of the duration of a transit, $D$, is

$$
d \ln D / d t=\frac{\dot{\omega} e \sin \left(f_{t}\right)}{\left(1+e \cos \left(f_{t}\right)\right)}\left\{1-\frac{b^{2}}{1-b^{2}}\right\} .
$$

where $b$ is the impact parameter and $f_{t}$ is the true anomaly at the time of transit. Note the interesting dependence on $b$. For $\dot{\omega} \delta t \sim 0.3 \mathrm{deg}$, we have that $\delta D \sim 5$ sec for a transit duration of $D \sim 0.15$ day. Kepler can measure the duration of transit to a precision $\sigma_{D} \sim 1.5 \mathrm{sec}$ in one year for a Jupiter-mass system with $P=5$ days orbiting a $V=12$ solar-type star and should therefore be capable of detecting changes in the transit duration $D$ due to GR within its mission for some eccentric, close-in systems.

\subsection{Time Between Primary and Secondary Transits}

If the time of the primary eclipse is given by $t_{1}$ and that of the secondary by $t_{2}$, the time difference between the two as compared to half a period $P, \Delta t \equiv t_{2}-t_{1}-0.5 P$, is given for central transits by:

$$
\Delta t=\frac{P}{\pi}\left[\frac{e \cos (\omega) \sqrt{1-e^{2}}}{\left(1-(e \sin \omega)^{2}\right)}+\arctan \left(\frac{e \cos \omega}{\sqrt{1-e^{2}}}\right)\right]
$$

(Sterne 1939). Uncertainties in $t_{2}$ will dominate the uncertainty in $\Delta_{t}$, so we assume $\sigma_{\Delta_{t}} \approx \sigma_{t_{2}} \sim 80 \mathrm{sec}$ (e.g., Deming et al. 2006; Charbonneau et al. 2008). Determining $\Delta t$ at two epochs separated by 10 years with a facility like Spitzer would detect the changes in $\Delta t$ due to GR with a significance $\gtrsim 4 \sigma$ in an eccentric system that precesses $\gtrsim 0.2$ deg in a decade if they have $\omega$ around $\pi / 2$ or $3 \pi / 2$.

$\dagger$ For stars we expect $k_{2, s} \lesssim 0.01$ (Claret \& Gimenez 1992), while for giant planets we expect $k_{2, p} \approx 0.25$ if we assume that their structure can be roughly described by a polytrope of index $n \approx 1$. 


\subsection{Radial Velocities}

Using simulations we find that radial velocity observations alone would be able to detect changes in $\omega$ induced by GR effects in scales of tens of years or less only for eccentric super-massive $\left(K \sim 1000 \mathrm{~m} \mathrm{sec}^{-1}\right)$ exoplanets orbiting close to a host star with a lowlevel of stellar jitter. For the detection to be statistically significant, on the order of 100 precise radial velocity observations $(\sigma \sim 2 \mathrm{~m} / \mathrm{s})$ are needed at each of two epochs separated by $\sim 20$ years.

\section{Detecting Terrestrial Mass Planets via Secular Changes in $\omega$.}

We briefly consider the detectability of $\dot{\omega}_{\text {perturber }}$ with Kepler using the change in $D$, which might complement the use of transittime variations (Agol et al. 2005; Holman \& Murray 2005) to infer the presence of terrestrial mass companions. We show in the Figure to the right the expected value of $\dot{\omega}_{\text {perturber }}$ as a function of $a_{2} / a$ assuming $a=0.05 \mathrm{AU}$, $M_{\star}=M_{\odot}$, and $f_{t}=0.5 \pi$ as solid lines, one for $M_{2}=M_{\oplus}$ and another for $M_{2}=10 M_{\oplus}$. The dashed lines mark the values of $\dot{\omega}_{\text {detect }}$ for $e=0.2$ and $e=0.5$, where $\dot{\omega}_{\text {detect }}$ is the value that Kepler could detect for a $V=12$ solartype star with a $P=5$ day planet. This figure shows that over a range of values of $\left(a_{2} / a\right) K e$ -

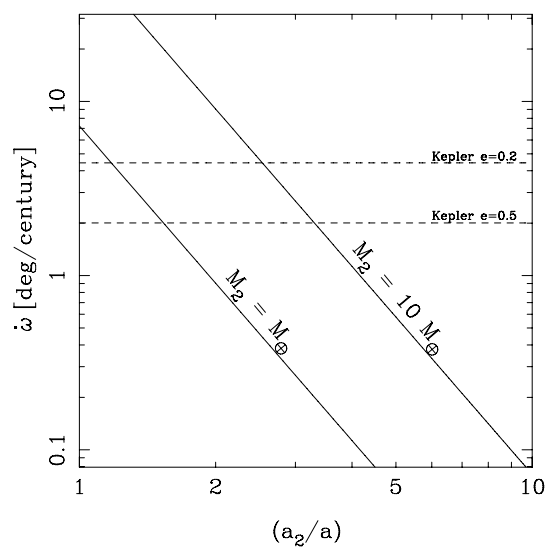

pler may be able to detect the presence of additional super-Earths $\left(M_{\oplus} \lesssim M \lesssim 10 M_{\oplus}\right)$ if the primary planet has a favorable impact parameter and true anomaly at the time of transit.

\section{References}

Agol, E., Steffen, J., Sari, R., \& Clarkson, W. 2005, MNRAS, 359, 567

Butler, R. P., Wright, J. T., Marcy, G. W., Fischer, D. A., Vogt, S. S., Tinney, C. G., Jones, H. R. A., Carter, B. D., Johnson, J. A., McCarthy, C., \& Penny, A. J. 2006, ApJ, 646, 505 Charbonneau, D., Knutson, H. A., Barman, T., Allen, L. E., Mayor, M., Megeath, S. T., Queloz, D., \& Udry, S. 2008, ArXiv e-prints arXiv:0802.0845

Claret, A. \& Gimenez, A. 1992, A\& AS, 96, 255

Deming, D., Harrington, J., Seager, S., \& Richardson, L. J. 2006, ApJ, 644, 560

Heyl, J. S. \& Gladman, B. J. 2007, MNRAS, 377, 1511

Holman, M. J. \& Murray, N. W. 2005, Science, 307, 1288

Jordán, A. \& Bakos, G. 2008, ApJ, in press (arXiv:0806.0630)

Miralda-Escudé, J. 2002, ApJ, 564, 1019

Murray, C. D. \& Dermott, S. F. 1999, Solar system dynamics (Cambridge: Cambridge University Press, 1999)

Pál, A. \& Kocsis, B. 2008, MNRAS, in press

Quataert, E. J., Kumar, P., \& Ao, C. O. 1996, ApJ, 463, 284

Smeyers, P. \& Willems, B. 2001, A\&A, 373, 173

Sterne, T. E. 1939, MNRAS, 99, 451

Wu, Y. \& Goldreich, P. 2002, ApJ, 564, 1024 